\title{
Perception of affective prosody in major depression: A link to executive functions?
}

\author{
JENNIFER UEKERMANN,${ }^{1}$ MONA ABDEL-HAMID, ${ }^{1}$ CAROLINE LEHMKÄMPER,${ }^{1}$ \\ WOLFGANG VOLLMOELLER, ${ }^{2}$ AND IRENE DAUM ${ }^{1}$ \\ ${ }^{1}$ Institute of Cognitive Neuroscience, Ruhr-University of Bochum, Bochum, Germany \\ ${ }^{2}$ Westfälisches Zentrum für Psychiatrie und Psychotherapie, Bochum, Germany
}

(Received May 9, 2007; Final Revision March 5, 2008; Accepted March 5, 2008)

\begin{abstract}
Major depression is associated with impairments of executive functions and affect perception deficits, both being linked to dysfunction of fronto-subcortical networks. So far, little is known about the relationship between cognitive and affective deficits in major depression. In the present investigation, affect perception and executive functions were assessed in 29 patients with a diagnosis of major depression (Dep) and 29 healthy controls (HC). Both groups were comparable on IQ, age, and gender distribution. Depressed patients showed deficits of perception of affective prosody, which were significantly related to inhibition, set shifting, and working memory. Our findings suggest a significant association between cognitive deficits and affect perception impairments in major depression, which may be of considerable clinical relevance and might be addressed in treatment approaches. Future studies are desirable to investigate the nature of the association in more detail. (JINS, 2008, 14, 552-561.)
\end{abstract}

Keywords: Depression, Affect perception, Executive, Social cognition, Theory of mind, Prosody

\section{INTRODUCTION}

Major depression is associated with cognitive impairments in the domains of memory and executive functions (Zakzanis et al., 1998; Kindermann et al., 2000) as well as interpersonal and social cognition problems (Levendosky et al., 1995).

Executive functions can be defined as "higher-level" functions involved in the control and regulation of "lower level" cognitive processes and the regulation of goal-directed behavior (Alvarez \& Emory, 2006). In a meta-analysis, Veiel (1997) found the largest differences between depressed patients and healthy controls on set shifting and inhibition tasks. In addition, planning and working memory impairments have been frequently observed in major depression (see DeBattista, 2005). Executive functions are of clinical relevance in the light of studies that have reported significant associations between executive dysfunction and relapse and recurrence of depression (Alexopoulos et al., 2000, 2002).

Interpersonal and social cognition problems in depression are reflected by reduced frequency of social inter-

Correspondence and reprint request to: Jennifer Uekermann, Institute of Cognitive Neuroscience, GAFO 05/607, Ruhr-University of Bochum, 44780 Bochum, Germany. E-mail: jennifer.uekermann@ ruhr-uni-bochum.de action (Gotlib \& Lee, 1989), reduced reward value of social interaction (Nezlek et al., 2000), and impaired theory of mind (Kerr et al., 2003; Inoue et al., 2006). Theory of mind refers to the ability to reason about mental states, to predict and to understand other people's behavior on the basis of their mental states (Premack \& Woodruff, 1978). An important aspect of theory of mind abilities is affect perception, the ability to decode mental states from tone of voice, body posture, or facial expression.

On the neuroanatomical level, functional impairments of fronto-subcortical circuits have been suggested to underlie these impairments (Brody et al., 2001; Campbell \& MacQueen, 2006). The dorsolateral frontal cortex, which projects to the dorsolateral head of the caudate nucleus has been linked to executive functions, whereas the ventromedial circuit, which includes projections from the anterior cingulate to the nucleus accumbens has been linked to motivation and social interaction (see Alvarez \& Emory, 2006). The orbitofrontal cortex projects to the ventromedial caudate nucleus and is involved in the mediation of social behavior (see Alvarez \& Emory, 2006). Mayberg et al. (1999) outlined reciprocal activation changes involving subgenual cingulate and right prefrontal cortex during transient and chronic changes in negative mood, which 
might mediate the link between mood and cognitive function in depression. A recent study by Siegle et al. (2007) reported enhanced amygdala reactivity during affective processing and reduced amygdala activation during executive function processing. Amygdala hyperreactivity is modulated by a common regulatory variant in the human serotonin transporter gene (Hariri et al., 2005).

The neural system underlying affective prosody comprehension may involve right-sided perisylvian regions that are organized in analogy to language areas of the left hemisphere (Ross, 1981). According to this model, expression of emotional prosody is dependent upon the homologue of Broca's area in the right inferior frontal cortex, whereas comprehension is believed to depend on the homologue of Wenicke's area in the right superior temporal region. Wildgruber et al. (2006) recently described three successive stages of emotional prosody perception. The first stage involves the extraction of suprasegmental acoustic information, the second stage the representation of meaningful suprasegmental acoustic sequences. The third stage entails the explicit evaluation of the emotional prosody. The first stage mainly recruits primary and higher order acoustic regions of the right hemisphere, the second stage posterior regions of the right superior temporal sulcus and the third stage is linked to bilateral processing in the inferior frontal cortex.

Studies of affect perception in major depression have mainly focused on the perception of facial stimuli. Mikhailova et al. (1996) presented pictures of sad, emotionally neutral, and happy faces on a computer screen randomly in the left or right visual hemifield. Depressed patients showed deficits in the identification of negative facial expression in the left and right hemifield and identification deficits for positive facial emotions in the left hemifield. The production of facial expressions was also found to be affected. In a study by Jaeger et al. (1986), depressed patients were less able than controls to produce emotional facial expressions, particularly positive ones. With regard to the clinical relevance of affect perception in depression, Persad and Polivy (1993) reported that depressed college students made more errors in recognizing facial expressions. In addition, they showed more freezing and tensing, that is, higher fear and depression reactions to these expressions. Judgment of negative emotions has been found to be related to depression severity and persistence (Hale, 1989). Impaired perception of sadness, rejection, or anger was associated with a less favorable course of depression (Bouhuys et al., 1996) and poor perception of negative emotions in faces was associated with relapse (Bouhuys et al., 1999). In a functional neuroimaging study by Canli et al. (2005), amygdala reactivity to emotional faces predicted improvement of clinical symptoms. These findings suggest that inappropriate reactions may be linked to maintenance or exacerbation of depression.

So far, little is known about the perception of affective prosody in major depression. In an early investigation, Murphy and Cutting (1990) presented neutral, angry, surprised, and sad sentences to depressed patients. Depressed patients showed a deficit in the identification of emotional tone when compared with healthy controls and made more errors when they had to detect sadness. Emerson et al. (1999) presented verbal statements to school-aged boys, half of whom had been classified as being depressed. Stimuli included simple declarative sentences in a happy, sad, angry, and neutral tone. On half of the trials, the emotional prosody of the sentences was congruent with the semantic content, on the remaining trials emotional prosody and content were incongruent. Depressed boys showed perception impairments for both congruent and incongruent affective prosody. Kan et al. (2004) presented semantically neutral sentences and short nonsense sentences, using tone to convey happiness, sadness, anger, fear, surprise, and disgust. Apart from a problem in identifying surprise, depressed subjects could adequately identify happiness, sadness, fear, and disgust.

The available data do not allow any firm conclusions as to whether affect perception impairments reflect a deficit in emotional perception per se or whether they can be attributed to impairments in cognitive domains, such as executive functions. Judging emotional prosody in spoken sentences requires maintenance of stimuli in working memory, inhibition of the task-irrelevant semantic meaning of the sentences, and/or successful switching between the semantic meaning and emotional prosody. Associations of these executive abilities with affect perception have been observed for different clinical conditions. In a study by Tompkins and Flowers (1985), three emotional perception tasks of differing complexity were administered to patients with right (RHD) or left (LHD) hemisphere damage. Whereas RHD patients were impaired on all tasks, the LHD group was selectively impaired on the third task, in which selection of affect category had to be made from an array of four alternatives. Breitenstein et al. (2001) reported that working memory was a significant predictor of prosody perception in patients suffering from Parkinson's disease. In addition, Bozikas et al. (2004) observed significant associations between affect perception and executive functions in schizophrenic patients.

In major depression, little is known about the relationship between cognitive and affect perception deficits. In a recent study by Langenecker et al. (2005), depressed women showed significant facial affect perception deficits and impairments of inhibitory control, which were unrelated. However, participants were outpatients and depression severity, as assessed by the Beck Depression Inventory, was relatively mild. In addition, the ability to interpret emotional prosody was not assessed.

To our knowledge, there are no studies to date which have addressed the potential contribution of executive deficits to impairments of affective prosody perception in major depression. This issue may be of considerable clinical relevance, given previous reports of a link between perception deficits and an unfavorable course of depression (see above). The aim of the present study was to assess the relationship between selected aspects of executive function (inhibition, 
set shifting, working memory) which might be particularly relevant for affect perception and the perception of emotional prosody in major depression. Based on previous reports of executive function impairment in depression and of significant associations between executive functions and affect perception, depressed patients were expected to show deficits in the perception of affective prosody which should be related to executive impairments.

\section{METHODS}

\section{Participants}

Fifty-eight subjects took part in the present investigation. All gave informed consent in accordance with the guidelines of the Declaration of Helsinki (Varga, 1984). The study was approved by the ethics committee of the Ruhr-University, Bochum, Germany. The clinical group (DEP) included 29 patients with a current diagnosis of major depression (14 men and 15 women). Major depression was diagnosed on the basis of a structured clinical interview according to DSM-IV ( $\mathrm{Sa}$ et al., 2003). Participants with major depression were inpatients in the Westfälisches Zentrum für Psychiatrie und Psychotherapie, Bochum. The healthy control group (HC) comprised 29 healthy controls (13 men and 16 women). A total of 13 patients had no previous depressive episode, 16 patients had a history of one previous episode. Mean disease duration was 18.07 months $(S D=11.21)$. Duration of current episode was 14.41 months $(S D=8.72)$ on average. Medication consisted of selective serotonin and noradrenalin reuptake inhibitors (Venflaxin, Escitalopram, Sertralin; $n=$ 6), selective noradrenergic and serotonergic antidepressants (Mirtazapin; $n=5$ ), tricyclic antidepressants (Trimipramin, Opipramol, Clomipramin; $\mathrm{n}=4)$, lithium $(n=2)$, and neuro- leptics (Olanzapin; $n=1$ ), and no medication $(n=17)$. Exclusion criteria were a history of neurological disorders, head trauma, anoxia, psychotic symptoms, stroke, learning disability, and addictive and psychiatric disorders (except depression in the DEP group). Healthy controls were recruited by advertisement and health status was assessed in an interview. As expected, the DEP group scored significantly higher on the Beck Depression Inventory (Beck, 1987) $[t(56)=$ $-12,49 ; p<.0001]$. Twenty-seven of the patients and controls also took part in a study of humor processing in major depression. The data are described elsewhere (Uekermann et al., 2008).

The two groups did not differ significantly in age or general intellectual status (IQ; both $p>.16$ ), as assessed by the Picture Completion and Similarities subtest of the short version of the Wechsler Adult Intelligence Scales (WIP; Dahl, 1986). The WIP is a short version based on the Wechsler Adult Intelligence Scales (WAIS; Wechsler, 1964) normed on German participants with a reliability of .94 and correlations with WAIS-scores of .92. There were no significant differences between the two subtests of the short version of the Wechsler Adult Intelligence Scales (both $p>$ .15). In addition, the proportion of males and females did not differ significantly between groups $\left(\chi^{2}=0,069 ; p=\right.$ .79). Demographic variables, affect and IQ estimates are presented in Table 1.

\section{Perception of Affective Prosody}

Perception of affective prosody was assessed by four subtests of the Tübingen Affect Battery (Breitenstein et al., 1996, 1998), a German adaptation of the Florida Affect Battery-Revised (Blonder et al., 1991). The internal consistency (Cronbach's Alpha) of the test battery is .97. All

Table 1. Demographic data, IQ, and percentage of correct responses for emotional prosody comprehension (means and standard errors) in the two groups

\begin{tabular}{|c|c|c|c|c|}
\hline & DEP & $\mathrm{HC}$ & $d f$ & $T$ \\
\hline$N$ & 29 & 29 & & \\
\hline Age & $37.00(1.62)$ & $39.07(2.35)$ & 56 & 0.72 \\
\hline Short version of the Wechsler Adult Intelligence Scales & $109.12(2.32)$ & $113.07(1.51)$ & 56 & -1.42 \\
\hline Beck Depression Inventory*** & $27.38(1.82)$ & $3.17(0.65)$ & 56 & 12.49 \\
\hline Naming neutral semantic content $* * *$ & $72.41(2.86)$ & $88.73(1.93)$ & 56 & -4.73 \\
\hline \multicolumn{5}{|l|}{ Naming congruent and incongruent semantic content } \\
\hline Congruent & $89.16(3.86)$ & $94.58(1.65)$ & 56 & -1.29 \\
\hline Incongruent $^{* * *}$ & $75.31(2.84)$ & $91.59(1.77)$ & 56 & -4.86 \\
\hline Matching of affective prosody to facial expression* & $83.44(2.55)$ & $93.10(1.30)$ & 56 & -3.37 \\
\hline Matching of facial expression to affective prosody* & $83.74(2.74)$ & $94.33(1.03)$ & 56 & -3.62 \\
\hline \multicolumn{5}{|l|}{ 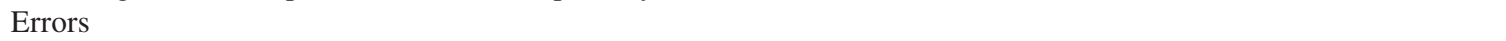 } \\
\hline Happiness*** & $6.28(0.69)$ & $2.45(0.51)$ & 56 & 4.46 \\
\hline Sadness & $1.86(0.30)$ & $1.21(0.22)$ & 56 & 1.73 \\
\hline Neutral* & $1.00(0.24)$ & $0.24(0.08)$ & 56 & 3.02 \\
\hline Anger* & $0.83(0.24)$ & $0.21(0.08)$ & 56 & 2.43 \\
\hline Fear*** & $5.72(0.80)$ & $1.86(0.42)$ & 56 & 4.29 \\
\hline
\end{tabular}

Note. $\mathrm{DEP}=$ depressed patients; $\mathrm{HC}=$ healthy controls. ${ }^{*} p<.05 ; * * p<.0001$ 
sentences of the Florida Affect Battery were translated to match the original version as closely as possible. The sentences were spoken by a professional actress. Stimuli represent the emotional categories "anger," "happiness," "fear," "sadness," and "neutral." Participants are asked to respond verbally or by pointing to a response card with the printed emotion labels. For each of the four subtests, correct responses (in \%) were assessed. In addition, error scores for the different affect categories were obtained (sums across the four subtests). Several practice items are administered in each subtest to ensure proper understanding of task instructions.

\section{Naming neutral semantic content}

The first subtest requires the naming of the affective prosody of sentences with neutral semantic content. In this subtest, 15 semantically neutral sentences (e.g., "The scarf is in the trunk") are administered. Participants have to name the affective prosody of the sentences. Naming neutral content involves holding the sentence in working memory.

\section{Naming congruent and incongruent semantic prosody}

This subtest involves the presentation of 32 sentences, half of which convey the same meaning in semantic content and emotional prosody (e.g., "She cried all day and night" spoken in a sad tone) and half of which involve a semantic content, which is incongruent with emotional prosody (e.g., "She cried all day and night" spoken in a happy tone). Participants are instructed to identify the emotional tone of voice and to ignore the semantic content. Processing of incongruent emotional prosody taps working memory, inhibition of semantic content, and switching between semantic content and emotional tone. Inefficient switching between semantic content and prosody and/or inefficient inhibition of semantic content would result in an increased errors score, because information from semantic content is not sufficient to complete the task.

\section{Matching of affective prosody to facial expression}

In this subtest, 15 semantically neutral sentences are presented (e.g., "The scarf is in the trunk.") in a tone, which represent one of the emotions described above. In addition, three faces are presented, depicting three different emotions. Participants are instructed to point to the face, which shows the same emotion as the emotional tone of the sentence. This subtest requires working memory, inhibition of emotional content of the distracter faces, as well as switching between faces and prosody.

\section{Matching of facial expression to affective prosody}

In this test, three semantically neutral sentences are presented in a tone, which convey an emotion (e.g., "The scarf is in the trunk" spoken in an angry voice). In addition, one emotional face is shown. Participants are instructed to select the sentence that involves the same emotion as the face. There are 15 trials. This subtest requires working memory, inhibition of emotional prosody of the distracter sentences, as well as switching between faces and prosody

\section{Measures of Executive Function}

\section{Inhibition}

Inhibition was assessed by a German adaptation of the Stroop Test (Bäumler, 1985). The Stroop Test consists of three subtests. The first condition ("Reading") requires reading aloud color words as fast as possible. In the second subtest ("Naming") participants are asked to name the color of colored bars. The third task ("Interference") involves naming the ink color of colored words printed in an incongruent color, such as "RED" in green ink, ignoring the word meaning. Dependent variables are the time needed to complete each subtest as well as the number of corrected and uncorrected errors in the interference condition. The time needed to complete the interference condition as well as errors in the interference condition were of particular interest within the context of this study.

\section{Working memory}

Working Memory was assessed by a German adaptation of the Letter-Number Sequencing task of the Wechsler Memory Scale (Wechsler, 1997). Sequences of letters and numbers are read aloud (e.g., 2-L-4) and have to be reproduced by repeating the numbers in ascending order followed by the letters in alphabetical order (2-4-L). Dependent variable was the number of correctly repeated sequences.

\section{Set shifting}

To assess set shifting, a German adaptation of the Trail Making Test (TMT; Reitan, 1992) was administered. In the first task, participants are instructed to draw lines to connect numbers printed on a page in ascending order (condition A). The second task involves alternating between numbers and letters in ascending order (e.g., 1-A-2-B). The time to complete the first and second subtests is assessed. Of particular relevance is condition B which reflects set shifting.

\section{RESULTS}

\section{Perception of Emotional Prosody}

The data for perception of affective prosody are shown in Table 1. Compared with the HC group, the DEP group showed fewer correct scores in the subtests "naming neutral semantic content" $[t(56)=4,73 ; p<.0001]$, "matching of affective prosody to facial expression" $[t(56)=3,37$; $p=.001]$ and "matching of facial expression to affective 
prosody" $[t(56)=3,62 ; p=.001]$. In addition, the DEP group performed more poorly on "naming incongruent semantic content" $[t(56)=4,86 ; p<.0001]$. There was no significant group difference for the "naming congruent semantic content" scores $(p=.20)$. The results for "naming congruent and incongruent affective prosody" are illustrated in Figure 1.

To explore potential differences between affect categories, analyses of variance (ANOVAs) with group (DEP versus HC) and affect categories ("anger," "happiness," "fear," "sadness," and "neutral") as factors were performed. Analysis yielded a significant group effect $[F(1,56)=23,62 ; p<$ $.0001]$ as well as a significant interaction $[F(2,224)=2,73$; $p<.0001]$. The DEP group made significantly more errors in all categories (all $p<.02$ ), except for stimuli depicting "sadness" $(p=.09)$.

\section{Executive Functions}

The results for the executive tests are described in Table 2.

\section{Working memory}

Analysis of the working memory task yielded a significantly lower score of DEP subjects when compared with $\mathrm{HC}[t(56)=4,36 ; p<.0001]$.

\section{Set shifting}

Repeated measures ANOVA for the TMT scores (A and B) yielded a significant group effect and a significant group $X$ condition interaction $[F(1,56)=7,16 ; p=.010]$. Separate analyses of TMTA and TMTB scores indicated that the DEP group was significantly slower in both conditions (both $p<$ .033). To further explore the interaction, difference scores (TMTB-TMTA) were analyzed. Analysis of the difference

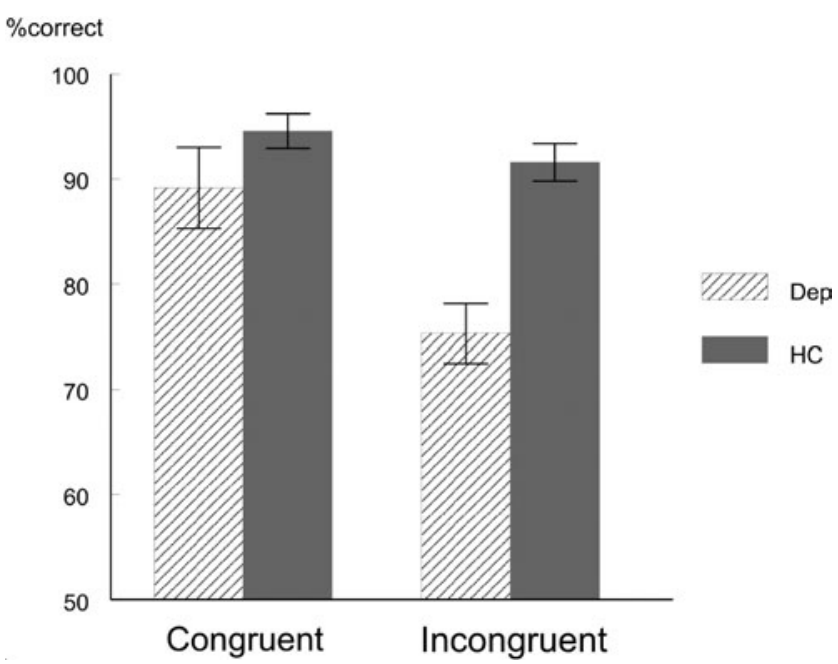

Fig. 1. Results (means and standard errors) for the two groups in the subtests "naming congruent and incongruent affective prosody." DEP = depressed patients; $\mathrm{HC}=$ healthy controls. scores yielded a significantly higher score of the DEP relative to the HC group $[t(56)=-2,68 ; p=.01]$.

\section{Inhibition}

Repeated measures ANOVA with condition and group as factors yielded a significant group effect $[F(1,56)=9,52$; $p=.003]$ and a significant group $\times$ condition interaction $[F(2,112)=4,24 ; p=.02]$. Subsequent $t$ tests indicated longer response times of the DEP relative to the $\mathrm{HC}$ group during reading $[t(56)=-4,04 ; p<.0001]$, naming $[t(56)=$ $-3,01 ; p=.004]$, and the interference condition $[t(56)=$ $-2,62 ; p=.01]$. In addition, analysis of difference scores (interference-naming) yielded a significant group effect $[t(56)=-2,05 ; p=.045]$, which was due to a significantly larger difference score of the DEP group in comparison with HC. The number of errors was significantly higher in the DEP group \{corrected errors: $[t(56)=2,56 ; p=.01]$; uncorrected errors: $[t(56)=4,56 ; p<.0001]\}$.

\section{Influence of Medication, Number of Episodes, and Potential Gender Effects}

Comparison of medicated and unmedicated depressed patients did not yield any significant group difference on any of the measures (all $p>.44$ ). To investigate the potential effect of the number of episodes, patients with a single previous episode were compared with patients without previous episodes. No significant effects were observed (all $p>.43$ ). In addition, analysis of gender differences for affect perception measures did not yield any significant group differences (all $p>.09$ ).

\section{Correlational Analyses}

To further investigate the relationship between executive functions and affect perception, correlational analyses between affect perception subtests and executive function measures (difference score of set shifting, difference score of inhibition, working memory) were carried out. Because one aim of this study was to investigate whether the observed findings are specific to the patient population or reflect a general phenomenon of complex prosody tasks, separate analyses were performed for the control and patient groups.

\section{Patient group}

The results for the patient group are shown in Table 3. As illustrated in Table 3, patients who had inhibition, working memory, and/or set shifting difficulties had also problems in naming neutral, incongruent semantic content, matching of affective prosody to facial expressions, and vice versa.

\section{Healthy control group}

The results for the healthy control group are shown in Table 4. As shown in Table 4, participants who had a higher 
Table 2. Results for the executive task (means and standard errors) in the two groups

\begin{tabular}{lrrrr}
\hline \hline & DEP & HC & $d f$ & $T$ \\
\hline Stroop test & & & & \\
$\quad$ Reading*** (time in sec) & $33.12(1.31)$ & $27.14(0.68)$ & 56 & 4.04 \\
$\quad$ Naming* (time in sec) & $47.65(2.39)$ & $39.68(1.12)$ & 56 & 3.01 \\
$\quad$ Interference* (time in sec) & $91.32(6.18)$ & $71.07(4.66)$ & 56 & 2.62 \\
$\quad$ Corrected errors** & $1.38(0.25)$ & $0.17(0.07)$ & 56 & 4.55 \\
$\quad$ Uncorrected errors* & $0.69(0.25)$ & $0.03(0.03)$ & 56 & 2.56 \\
Trail Making Test A* (time in sec) & $33.28(2.33)$ & $26.37(1.52)$ & 56 & 2.48 \\
Trail Making Test B* (time in sec) & $85.22(9.23)$ & $55.93(3.66)$ & 56 & 2.95 \\
Number-Letter task*** & $6.28(0.39)$ & $8.38(0.28)$ & 56 & -4.36 \\
$\quad$ (number of correctly repeated sequences) & & & & \\
\hline \hline
\end{tabular}

Note. $\mathrm{DEP}=$ depressed patients; $\mathrm{HC}=$ healthy controls. $* p<.05 ; * * * p<.0001$.

difference scores in the Stroop test (inhibition-naming) had more difficulties in naming congruent semantic content.

\section{DISCUSSION}

To our knowledge, this is the first study, in which the potential contribution of executive dysfunction to deficits of affective prosody comprehension was investigated in major depression. Patients with major depression were expected to show affect perception impairments, which might be linked to executive functions. The findings of the prosody perception task indicate that patients with major depression selected fewer correct solutions for "naming neutral semantic content," "naming incongruent semantic content," "matching of affective prosody to facial expression," and "matching of facial expression to affective prosody." Depressed patients, however, performed well when prosody with congruent semantic content had to be named, which may be due to the fact that working memory, inhibition of the task-irrelevant semantic meaning of the sentences, and efficient switching between content and prosody are not critical for successful completion of the task (see below). Depressed patients were impaired across all major affect categories, with the exception of stimuli reflecting sadness. Similarly, they showed deficits across a range of different executive function subcomponents such as set shifting, working memory, as well as inhibition. The observed deficits cannot be attributed to medication effects because there was no significant difference in the profile of medicated and unmedicated depressed patients.

Taken together, the results suggest that depressed patients suffer from affective prosody comprehension deficits, which may be influenced by executive functions. The observed impairments are in line with previous studies, which have reported similar deficits (Murphy \& Cutting, 1990; Emerson et al., 1999), although Kan et al. (2004) found contradictory results (see below). The incongruent condition of the Tübingen Affect Battery is of particular relevance because it is assumed to tap resistance to distraction in the face of conflicting information (Bowers et al., 1987). However, the fact that depressed patients did also show deficits when semantic content was neutral, suggests that interference alone cannot account for the problems they experience. In the cross-modal matching tasks, processing of faces is also involved and sentences are emotionally neutral in semantic content. Thus the results may be interpreted as a more fundamental impairment in affective prosody comprehension, which appears to be masked when other cues are present to aid the identification the meaning of affective prosody. The finding that depressed patients had higher error scores for all categories except for stimuli depicting "sadness" is consistent with the negative bias of depressed patients, which has been frequently reported (Beck et al., 1979; Suslow et al., 2001; Kan et al., 2004). This information processing bias could lead to selective or focused attention to sad stimuli, leading to enhanced processing of such stimuli.

Table 3. Results for the correlational analyses for the patient group

\begin{tabular}{llllll}
\hline \hline & \multicolumn{1}{c}{ NNSC } & NCSC & \multicolumn{1}{c}{ NISC } & MoAPFE & MoFEAP \\
\hline WM & $\mathrm{r}=.45^{*}$ & $\mathrm{r}=.05$ & $\mathrm{r}=.64 * * *$ & $\mathrm{r}=.39 *$ & $\mathrm{r}=.47 *$ \\
INH & $\mathrm{r}=-.63^{* * * *}$ & $\mathrm{r}=-.15$ & $\mathrm{r}=-.65^{* * * *}$ & $\mathrm{r}=-.57 * *$ & $\mathrm{r}=-.53^{* *}$ \\
SET & $\mathrm{r}=-.55^{* *}$ & $\mathrm{r}=-.02$ & $\mathrm{r}=-.56^{* *}$ & $\mathrm{r}=-.64 * * *$ & $\mathrm{r}=-.53^{* *}$ \\
\hline
\end{tabular}

Note. $\mathrm{WM}=$ working memory $; \mathrm{INH}=$ inhibition; $\mathrm{SET}=$ Set Shifting; $\mathrm{NNSC}=$ Naming neutral semantic content; NCSC $=$ Naming congruent semantic content; NISC $=$ Naming incongruent semantic content; MoAPFE = Matching of affective prosody to facial expressions; MoFEAP = Matching of facial expressions to affective prosody. ${ }^{*} p<.05$; ${ }^{*} p<.01 ; * * * p<.0001$. 
Table 4. Results for the correlational analyses for the healthy control group

\begin{tabular}{llllll}
\hline \hline & NNSC & NCSC & NISC & MoAPFE & MoFEAP \\
\hline WM & $\mathrm{r}=-.16$ & $\mathrm{r}=.04$ & $\mathrm{r}=-.05$ & $\mathrm{r}=-.10$ & $\mathrm{r}=.17$ \\
INH & $\mathrm{r}=-.13$ & $\mathrm{r}=-.45^{*}$ & $\mathrm{r}=-.20$ & $\mathrm{r}=-.06$ & $\mathrm{r}=-.16$ \\
SET & $\mathrm{r}=-.22$ & $\mathrm{r}=.18$ & $\mathrm{r}=-.10$ & $\mathrm{r}=.17$ & $\mathrm{r}=-.21$ \\
\hline \hline
\end{tabular}

Note $. \mathrm{WM}=$ working memory; $\mathrm{INH}=$ inhibition; $\mathrm{SET}=$ Set Shifting; $\mathrm{NNSC}=$ Naming neutral semantic content; NCSC $=$ Naming congruent semantic content; NISC $=$ Naming incongruent semantic content; MoAPFE $=$ Matching of affective prosody to facial expressions; MoFEAP $=$ Matching of facial expressions to affective prosody. ${ }^{*} p<.05 ; * * p<.01 ; * * p<.0001$.

The present findings suggest that executive dysfunction may contribute to the impairments. Correlational analyses revealed significant associations between prosody comprehension, set shifting, inhibition, as well as working memory. We assume that working memory, inhibition, and set shifting are not critical for the identification of congruent emotional prosody. For the identification of incongruent emotional prosody, the semantic content and emotional prosody have to be reanalyzed. We assume that this reanalysis does require set shifting, inhibition, and working memory. Further studies are necessary to investigate the validity of the proposed model and to allow conclusions concerning the potential differential contribution of executive subcomponents to the comprehension of affective prosody. The proposed relationships between executive functions and affect perception are illustrated in Figure 2.

Depressed patients did not show any deficits when congruent affective prosody had to be named. The identifica- tion of congruent affective prosody is not critically dependent upon working memory, inhibition, and set shifting abilities. The contradictory results by Kan et al. (2004) may also be explained in the context of executive functions. Kan et al. (2004) presented semantically neutral sentences (such as "Good morning") and short nonsense sentences using tone to convey happiness, sadness, anger, fear, surprise, and disgust. The performance of depressed patients was intact, presumably because nonsense and/or very short sentences did not recruit working memory, inhibitory abilities, and task switching.

It may be argued that the associations between affect perception deficits and executive impairments might reflect a common underlying deficit resulting in dysfunction of both systems, which are generally independent of each other. This view would be consistent with a recent meta-analysis by Fusar-Poli et al. (2006), in which acute trytophan depletion was found to modulate cortical activity in areas that are

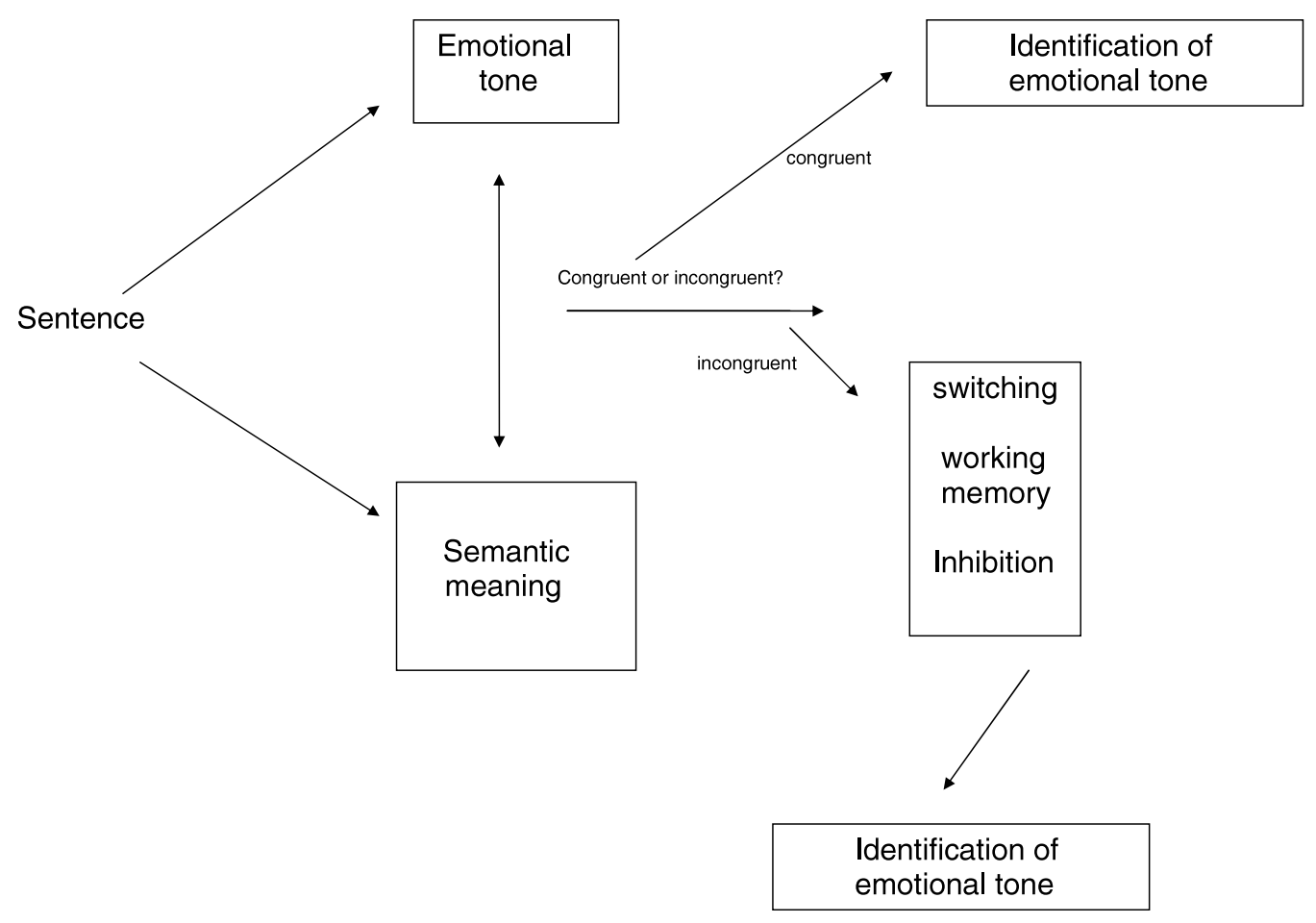

Fig. 2. Potential contribution of executive functions to affective prosody perception. 
implicated in executive functions (dorsolateral prefrontal cortex) and emotional processing (orbitofrontal, cingulate, and subcortical regions). Further studies are clearly desirable to investigate the nature of the observed associations between executive and affect perception deficits in more detail.

The findings of the present investigation are inconsistent with the findings by Langenecker et al. (2005). The authors reported executive impairments and deficits in the perception of emotional faces that were unrelated in depressed patients. The inconsistency with respect to our findings may be at least partly due to differences in depression severity and task complexity. Emotional prosody is more demanding than facial affect processing, as accuracy scores for the perception of emotional faces are usually higher than prosody accuracy scores (Johnstone \& Scherer, 2000). In addition, depressed women in the study by Langenecker et al. (2005) were not inpatients and depression levels were relatively low.

With respect to neural correlates of emotion perception (Phillips et al., 2003), the ventral system is involved in the identification of the emotional significance of stimuli and the production of emotional behavior and states. The dorsal system is engaged in executive functions and effortful regulation of emotional states. In addition, a reciprocal functional relationship between these two neural systems is assumed, which may serve as the basis of cognitiveaffective interactions. The present results would be consistent with a dysfunction of the interaction of these systems in major depression. Executive deficits might be linked to dysfunction of the dorsal system and affect perception deficits to dysfunction of the ventral system.

An important issue is whether the observed deficits of depressed patients may simply result from low effort in the patients. In the light of the finding that the groups did not differ significantly on many measures such as picture completion, similarities and naming congruent semantic content, reduced effort, and low motivation are not a likely explanation for the observed impairment pattern, although this possibility cannot be completely ruled out. In addition, the observed impairments could be due to ruminative thoughts, which have been frequently reported in major depression (Nolen-Hoeksema, 2000), and the investigation of ruminative thoughts to cognitive and affective processing is clearly a matter of future research interest.

Several limitations of the present study have to be considered. First, the sample sizes are relatively small and the findings should thus be interpreted with caution. Second, more basic cognitive abilities have not been comprehensively assessed. It is thus possible that the observed findings at least partly reflect general perceptual-motor deficits rather than impairments of affect perception and executive functions, although it should be pointed out that both groups were comparable on the similarities and picture completion subtests of the WIP as well as on naming congruent semantic content. However, a more comprehensive neuropsychological test battery would have been desirable but could no be added to clinical routine assessment because of time constraints. It should also be noted that correlational analyses of course only reflect associations and not causal links.

Affect perception deficits are doubtlessly of considerable clinical relevance. They can contribute to interpersonal problems and negatively affect the therapeutic process in major depression. Although the influence of prosody perception deficits on the course of depression is as yet unknown, there is evidence for such a link from studies of emotional face perception (Bouhuys et al., 1996; Canli et al., 2005; Hale, 1989; Persad \& Polivy, 1993). The findings of these studies suggest that inappropriate responses may be associated with maintenance or increase of depression. However, the observed relationship between affect perception and clinical outcome may be also due to the correlation between affect perception and executive deficits. Executive deficits have previously been shown to be associated with relapse and recurrence of depression (Alexopoulos et al., 2000, 2002). Prosody perception deficits as well as executive deficits should thus be considered in therapeutical intervention strategies.

In summary, the present investigation suggests that patients with major depression suffer from deficits in the perception of affective prosody, which are related to reduced set shifting and working memory problems. In the light of the potential clinical significance of affect perception in major depression, assessment of such problems should be taken into consideration in the therapeutic setting. Future studies are clearly desirable to investigate the nature of the association in further detail.

\section{ACKNOWLEDGMENTS}

We disclose any financial or other relationships that could be interpreted as a conflict of interest including those (a) with manufacturer(s) of any commercial products(s) and/or provider(s) of commercial services and (b) with any commercial support of the research reported in the manuscript submitted for publication. There are no sources of financial support.

\section{REFERENCES}

Alexopoulos, G.S., Kiosses, D.N., Klimstra, S., Kalayam, B., \& Bruce, M.L. (2002). Clinical presentation of the "depressionexecutive dysfunction syndrome" of late life. American Journal of Geriatric Psychiatry, 10, 98-106.

Alexopoulos, G.S., Meyers, B.S., Young, R.C., Kalayam, B., Kakuma, T., Gabrielle, M., Sirey, J.A., \& Hull, J. (2000). Executive dysfunction and long-term outcomes of geriatric depression. Archives of General Psychiatry, 57, 285-290.

Alvarez, J.A. \& Emory, E. (2006). Executive function and the frontal lobes: A meta-analytic review. Neuropsychological Review, 16, 17-42.

Bäumler, G. (1985). Farbe-Wort-Interferenztest (FWIT) nach J. Stroop (Colour-Word-Interference Test according to J. Stroop). Göttingen, Germany: Hogrefe.

Beck, A.T. (1987). Beck Depression Inventory. San Antonio, TX: The Psychological Corporation. 
Beck, A.T., Rush, A.J., Shaw, B.F., \& Emery, G. (1979). Cognitive Therapy of Depression. New York: Guilford Press.

Blonder, L.X., Bowers, D., \& Heilman, K.M. (1991). The role of the right hemisphere in emotional communication. Brain, 114, 1115-1127.

Bouhuys, A.L., Geerts, E., \& Gordijn, M.C. (1999). Depressed patients' perceptions of facial emotions in depressed and remitted states are associated with relapse: A longitudinal study. Journal of Nervous and Mental Diseases, 187, 595-602.

Bouhuys, A.L., Geerts, E., Mersch, P.P., \& Jenner, J.A. (1996). Nonverbal interpersonal sensitivity and persistence of depression: Perception of emotions in schematic faces. Psychiatry Research, 64, 193-203.

Bowers, D., Coslett, H.B., Bauer, R.M., Speedie, L.J., \& Heilman, K.M. (1987). Comprehension of emotional prosody following unilateral hemispheric lesions: Processing defect versus distraction defect. Neuropsychologia, 25, 317-328.

Bozikas, V.P., Kosmidis, M.H., Anezoulaki, D., Giannakou, M., \& Karavatos, A. (2004). Relationship of affect recognition with psychopathology and cognitive performance in schizophrenia. Journal of the International Neuropsychological Society, 10, 549-558.

Breitenstein, C., Daum, I., \& Ackermann, H. (1998). Emotional processing following cortical and subcortical brain damage: Contribution of the frontal-striatal circuitry. Behavioral Neurology, 11, 29-42.

Breitenstein, C., Daum, I., Ackermann, H., Lütgehetmann, R., \& Müller, E. (1996). Erfassung der Emotionswahrnehmung bei zentralnervösen Läsionen und Erkrankungen: Psychometrische Gütekriterien der "Tübinger Affekt Batterie." Neurologie \& Rehabilitation, 2, 93-101.

Breitenstein, C., Van Lancker, D., Daum, I., \& Waters, C.H. (2001). Impaired perception of vocal emotions in Parkinson's disease: Influence of speech time processing and executive functioning. Brain and Cognition, 45, 277-314.

Brody, A.L., Barsom, M.W., Bota, R.G., \& Saxena, S. (2001). Prefrontal-subcortical and limbic circuit mediation of major depressive disorder. Seminars in Clinical Neuropsychiatry, 6, 102-112.

Campbell, S. \& MacQueen, G. (2006). An update on regional brain volume differences associated with mood disorders. Current Opinion in Psychiatry, 19, 25-33.

Canli, T., Cooney, R.E., Goldin, P., Shah, M., Sivers, H., Thomason, M.E., Whitfield-Gabrieli, S., Gabrieli, J.D., \& Gotlib, I.H. (2005). Amygdala reactivity to emotional faces predicts improvement in major depression. Neuroreport, 16, 1267-1270.

Dahl, G. (1986). Reduzierter Wechlser Intelligenztest (Short version of the Wechsler Intelligence Test). Hain, Germany: Meisenheim.

DeBattista, C. (2005). Executive dysfunction in major depressive disorder. Expert Review of Neurotherapeutics, 5, 79-83.

Emerson, C.S., Harrison, D.W., \& Everhart, D.E. (1999). Investigation of receptive affective prosodic ability in school-aged boys with and without depression. Neuropsychiatry, Neuropsychology, and Behavioral Neurology, 12, 102-109.

Fusar-Poli, P., Allen, P., McGuire, P., Placentino, A., Cortesi, M., \& Perez, J. (2006). Neuroimaging and electrophysiological studies of the effects of acute tryptophan depletion: A systematic review of the literature. Psychopharmacology, 188, 131-143.

Gotlib, I.H. \& Lee, C.M. (1989). The social functioning of depressed patients: A longitudinal assessment. Journal of Social and Clinical Psychology, 8, 223-237.
Hale, W.W. (1989). Judgment of facial expressions and depression persistence. Psychiatry Research, 80, 265-274.

Hariri, A.R., Drabant, E.M., Munoz, K.E., Kolachana, B.S., Mattay, V.S., \& Egan, M.F. (2005). Weinberger DR. A susceptibility gene for affective disorders and the response of the human amygdala. Archives of General Psychiatry, 62, 146-152.

Inoue, Y., Tonooka, Y., Yamada, K., \& Kanba, S. (2006). Deficiency of theory of mind in patients with remitted mood disorder. Journal of Affective Disorders, 82, 403-409.

Jaeger, J., Borod, J.C., \& Peselow, E. (1986). Facial expressions of positive and negative emotions in patients with unipolar depression. Journal of Affective Disorders, 11, 43-50.

Johnstone, T. \& Scherer, K.R. (2000). Vocal communication of emotion. In M. Lewis \& J.M. Haviland-Jones (Eds.), Handbook of Emotions. New York: The Guilford Press.

Kan, Y., Mimura, M., Kamijima, K., \& Kawamura, M. (2004). Recognition of emotion from moving facial and prosodic stimuli in depressed patients. Journal of the International Neuropsychological Society, 75, 1667-1671.

Kerr, N., Dunbar, R.I., \& Bentall, R.P. (2003). Theory of mind deficits in bipolar affective disorder. Journal of Affective Disorders, 73, 253-259.

Kindermann, S.S., Kalayam, B., Brown, G.G., Burdick, K.E., \& Alexopoulos, G.S. (2000). Executive functions and P300 latency in elderly depressed patients and control subjects. American Journal of Geriatric Psychiatry, 8, 57-65.

Langenecker, S.A., Bieliauskas, L.A., Rapport, L.J., Zubieta, J.K., Wilde, E.A., \& Berent, S. (2005). Face emotion perception and executive functioning deficits in depression. Journal of Clinical and Experimental Neuropsychology, 27, 320-333.

Levendosky, A.A., Okun, A., \& Parker, J.G. (1995). Depression and maltreatment as predictors of social competence and social problem-solving skills in school-age children. Child Abuse \& Neglect, 19, 1183-1195.

Mayberg, H.S., Liotti, M., Brannan, S.K., McGinnis, S., Mahurin, R.K., Jerabek, P.A., Silva, J.A., Tehell, J.L., Martin, C.C., Lancaser, J.L., \& Fox, P.T. (1999). Reciprocal limbic-cortical function and negative mood: Converging PET findings in depression and normal sadness. American Journal of Psychiatry, 156, 675682.

Mikhailova, E.S., Vladimirova, T.V., Iznak, A.F., Tsusulkovskaya, E.J., \& Sushko, N.V. (1996). Abnormal recognition of facial expression of emotions in depressed patients with major depression disorder and schizotypal personality disorder. Biological Psychiatry, 40, 697-705.

Murphy, D. \& Cutting, J. (1990). Prosodic comprehension and expression in schizophrenia. Journal of the International Neuropsychological Society, 53, 727-730.

Nezlek, J.B., Hampton, C.P., \& Shean, G.D. (2000). Clinical depression and day-to-day social interaction in a community sample. Journal of Abnormal Psychology, 109, 11-19.

Nolen-Hoeksema, S. (2000). The role of rumination in depressive disorders and mixed anxiety/depressive symptoms. Journal of Abnormal Psychology, 109, 504-511.

Persad, S.M. \& Polivy, J. (1993). Differences between depressed and nondepressed individuals in the recognition of and response to facial emotional cues. Journal of Abnormal Psychology, 102, $358-368$

Phillips, M.L., Drevets, W.C., Rauch, S.L., \& Lane, R. (2003). Neurobiology of emotion perception I: The neural basis of normal emotion perception. Biological Psychiatry, 54, $504-514$. 
Premack, D. \& Woodruff, G. (1978). Does the chimpanzee have a theory of mind? Behavioral and Brain Sciences, 4, 515-526.

Reitan, R.M. (1992). Trail Making Test: Manual and Administration for Scoring. South Tucson, AZ: Reitan Neuropsychology Laboratory.

Ross, E.D. (1981). The aprosodias. Functional-anatomic organization of the affective components of language in the right hemisphere. Archives of Neurology, 38, 561-569.

Saß, H., Wittchen, H.U., \& Zaudig, M. (2003). Diagnostisches und statistisches Manual psychischer Störungen (DSM-IV) (Diagnostical and Statistical Manual of Mental Disorders). Göttingen, Germany: Hogrefe.

Siegle, G.J., Thompson, W., Carter, C.S., Steinhauer, S.R., \& Thase, M.E. (2007). Increased amygdala and decreased dorsolateral prefrontal BOLD responses in unipolar depression: Related and independent features. Biological Psychiatry, 61, 198-209.

Suslow, T., Junghanns, K., \& Arolt, V. (2001). Detection of facial expressions of emotions in depression. Perceptual Motor Skills, 92, 857-868.

Tompkins, C.A. \& Flowers, C.R. (1985). Perception of emotional intonation by brain-damaged adults: The influence of task processing levels. Journal of Speech and Hearing Research, 28, 527-538.
Uekermann, J., Channon, S., Lehmkämper, C., Abdel-Hamid, M., Vollmoeller, W., \& Daum, I. (2008). Executive function, mentalizing and humor in major depression. Journal of the International Neuropsychological Society, 14, 55-62.

Varga, A.C. (1984). Declaration of Helsinki. (Adopted by the 18th World medical Assembly in Helsinki, Finland, and revised by the 29th World Assembly in Tokyo, 1975). In The Main Issue in Bioethics, revised edition. New York: Paulist Press.

Veiel, H.O. (1997). A preliminary profile of neuropsychological deficits associated with major depression. Journal of Clinical and Experimental Neuropsychology, 19, 587-603.

Wechsler, D. (1964). Die Messung der Intelligenz Erwachsener: Textband zum Hamburg-Wechsler-Intelligenztest für Erwachsene. Bern, Germany: Huber.

Wechsler, D. (1997). Wechsler Memory Scale-3rd Edition. San Antonio, Texas: Harcourt Assessment.

Wildgruber, D., Ackermann, H., Kreifelds, B., \& Ethofes, T. (2006). Cerebral processing of linguistic and emotional prosody: fMRI studies. Progress in Brain Research, 156, 249-268.

Zakzanis, K.K., Leach, L., \& Kaplan, E. (1998). On the nature and pattern of neurocognitive function in major depressive disorder. Neuropsychiatry, Neuropsychology, and Behavioral Neurology, 11, 111-119. 